\title{
SOFT TISSUES FINITE ELEMENT MODELING AND CALIBRATION OF THE MATERIAL PROPERTIES IN THE CONTEXT OF COMPUTER ASSISTED MEDICAL INTERVENTIONS
}

Yohan Payan, TIMC-IMAG Laboratory, Univ. Grenoble Alpes \& CNRS, F-38000 Grenoble, France. Phone : +334565200 01, Fax :+334565200 55, Email : Yohan.Payan@imag.fr

Key words: Finite Element modeling; Computer assisted surgical planning; constitutive law.

\begin{abstract}
This chapter aims at illustrating how patient-specific models of human organs / soft tissues can be implemented into Finite Element (FE) packages. First is addressed the question of the generation of patient-specific FE models compatible with the clinical constraints. Then is discussed the calibration of the material properties, with choices that should be done between calibrations based on ex vivo or in vivo tissues loadings. The example of computer assisted maxillofacial surgery is addressed and results based on patients' data are provided.
\end{abstract}

\section{INTRODUCTION}

Computer-Assisted Medical Interventions (CAMI) is now a mature domain, with pioneering works dating from the 80's. Researchers, clinicians and industrial partners have developed CAMI applications by building links with classical domains such as Computer Science, Robotics, Image Processing and Mathematics. The connection to Biomechanics is more recent and began with the study of bony structures. This was motivated by the fact that the pioneering CAMI devices were dedicated to orthopedics, where bones are the main anatomical structures of interest and were assumed to be non-deformable with a 3D shape easily reconstructible from Computed Tomography (CT) imaging. The main idea for such biomechanical modeling was to define a theoretical and numerical framework that provided information about the mechanics of the bones after a clinical treatment or a surgical intervention. For example, a patient-specific Finite Element (FE) model of the femur could be designed to estimate the internal stresses generated by a hip prosthesis and therefore to help limit fracture risks. In these continuous biomechanical models, bones were usually considered as linear elastic material that underwent small deformations, which permitted easy calculation of numerical solutions. 
More recently, CAMI has addressed a larger spectrum of clinical domains such as cardiology, neurosurgery, maxillofacial surgery, urology or abdominal surgery. For these applications, biomechanics faces a new challenge since the involved tissues are required to move and be deformed by stresses generated by clinical actions. Moreover, soft tissues are difficult to model accurately since they typically exhibit complex, patient-specific, time dependent, non-linear, inhomogeneous and anisotropic behaviors. Most of the corresponding biomechanical models need to include large deformation effects and visco-hyperelastic constitutive laws. Such models are very computationally demanding which explain why most of them are limited to pre-operative use, since the simulations often require many minutes or hours to compute.

This chapter aims at illustrating soft tissues modeling in the context of maxillofacial surgery where Finite Element models of the facial tissues have to be developed to predict the impact on facial aesthetics depending on the bone repositioning maxillofacial surgery plan. The following questions will be addressed (1) "How to generate a patient-specific Finite Element model in a framework compatible with the clinical constraints?" and (2) "How to calibrate the material properties for the modeled soft tissues?"

\section{COMPUTER ASSISTED ORTHOGNATHIC SURGERY}

\subsection{Clinical context}

Orthognathic surgery (the "surgery to create straight jaws", see Richter et al. (1998)) involves a wide variety of surgical procedures performed to reposition maxilla, mandible and the dento-alveolar segments to achieve facial and occlusal balance. This may be necessary due to congenital abnormalities, growth disturbances or trauma. Such corrections are largely achieved by osteotomies, surgical techniques by which parts of the jaw(s) are cut to create separate fragments, which can then be moved to new positions while preserving their blood supply (figure 1a). Correction of these abnormalities generally normalizes patients' dental occlusion and temporo-mandibular joint function, and results in improvement in functions such as chewing, speaking and breathing, while often enhancing facial aesthetics.

A model of the patient face used to simulate the morphological modifications following bone repositioning could greatly improve the planning of the intervention, for both the surgeon and the patient. 


\subsection{Patient-specific biomechanical model of the human face}

Various models of the face soft tissue were proposed in the literature. Whereas the first works were based on discrete models such as mass-spring models (Delingette et al., 1994 ; Waters, 1996 ; Keeve et al., 1996 ; Teschner et al., 1999 ; Barré et al., 2000), many continuous Finite Element models were then proposed in the literature (Koch et al., 1996 ; Keeve et al., 1998 ; Koch et al., 1999 ; Zachow et al., 2000 ; Gladilin et al., 2001 ; Teschner, 2001 ; Chabanas et al., 2003 ; Mollemans et al., 2007).

Building a Finite Element model of an organ requires an accurate observation of the anatomy of that organ with a description of the various structures that constitute the organ. In particular, a focus should be given to the mechanical differences that can be observed in terms of constitutive behaviors, from one sub-structure to another one. Facial tissues are composed of a complex interweaving of muscular fibers, fat, glands and mucosa (Rouviere and Delmas, 1997). The facial skin structure is basically made of three layers: the epidermis, the dermis and the hypo-dermis. The epidermis is a superficial $0.1 \mathrm{~mm}$ thick layer, mainly composed of dead cells. The underlying dermis layer, which is much thicker $(0.5-4 \mathrm{~mm})$, is composed of elastin fibers mainly responsible for the global elastic behaviour of the skin. Finally, the hypodermis layer, mainly fat tissues and mucosa, can slightly slide over the bones of the skull and jaw. The muscular structure (mostly flat muscles contained in the hypodermis layer) that connects these skin layers to the skull is extremely complex, with insertion points, orientations and interweaving fibers allowing great facial dexterity (mimics, expressions, lip gestures for speech production).

Our group has developed a "generic" 3D Finite Element face model implemented in the ANSYS software (Chabanas 2002 ; Chabanas et al., 2003 ; Nazari et al., 2010). The volume defined by the facial tissues located between the skull and the external skin surface of the face was manually meshed, as regularly as possible, with an 8 nodes hexahedra-dominant mesh representing the three layers of dermis and sub-dermis tissues with muscles courses identified inside the corresponding

elements (figure 1b). The mesh is symmetrical with respect to the mid-sagittal plane. Contacts are added to formulate inter-lip and lip/teeth collisions.

In the framework of computer-assisted maxillofacial surgery, a model of each patient is required. A 3D mesh adapted to each patient's morphology must therefore be defined. Most of the existing Finite Element face models are built from patient CT scans using automatic meshing methods. However, these methods usually lack robustness because of the complex geometry and topology of the face soft tissues, and the time needed to generate such meshes is generally several days, which is not compatible with clinical use. In addition, these models are limited in terms of biomechanical modeling. Indeed, the unstructured organization of the elements does not allow one anatomical 
structure to be distinguished from another within the mesh. The face soft tissues are thus modeled as a single entity, without distinctions between dermis layers, fat, muscles and mucosa. To overcome these limitations, our group has proposed a methodology called the Mesh-Match-and-Repair algorithm (Couteau et al., 2000 ; Bucki et al., 2010) consisting first, in manually defining a structured 3D mesh, in order to build one "generic" model of the face. Emphasis is given to the design of the generic mesh, so that the elements inside the mesh can be associated to anatomical entities (dermis layers, fat, muscles, mucosa...). Specific mechanical properties can therefore be explicitly given to such substructures. The next step of our methodology consists of matching the generic model to each patient's morphology, using an automatic elastic registration method: a structured Finite Element model of each patient is then automatically generated (figure 1c).

Figure 1 illustrates an example of the predictions provided by a Finite Element model adapted to the geometry of a patient. The bone repositioning planned by the surgeon (figure 1a) is put as an input to the model by imposing the corresponding displacements to the nodes that are supposed to be attached to the mandible, chin and maxillary segments (figure 1d). The results provided by the simulation can therefore be compared with the actual outcome of the surgery (figure 1e).
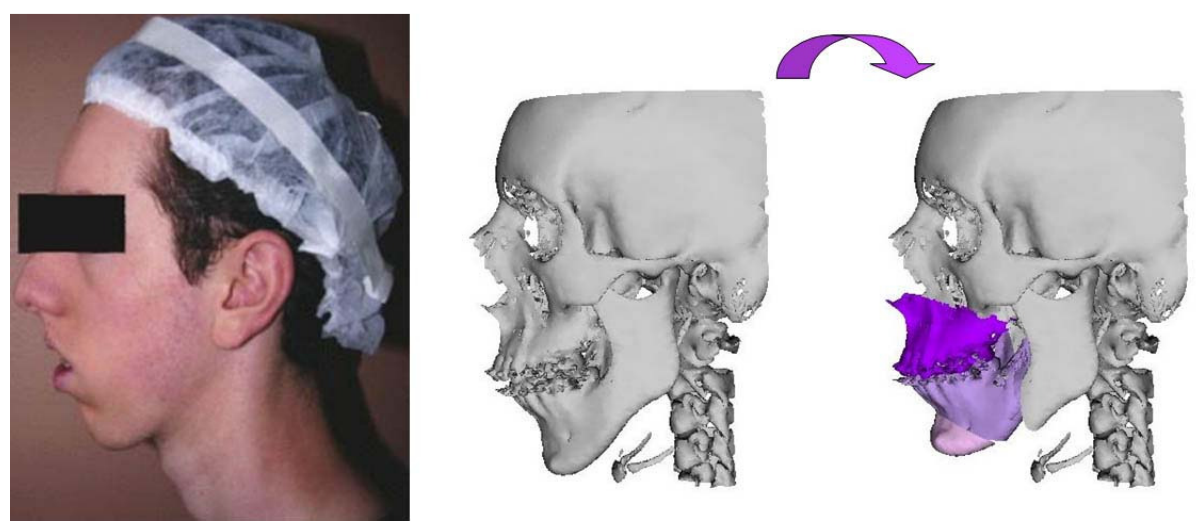

Figure 1a: A patient with a mandibular dysmorphosis (left) and the planned bone cuttings (right)
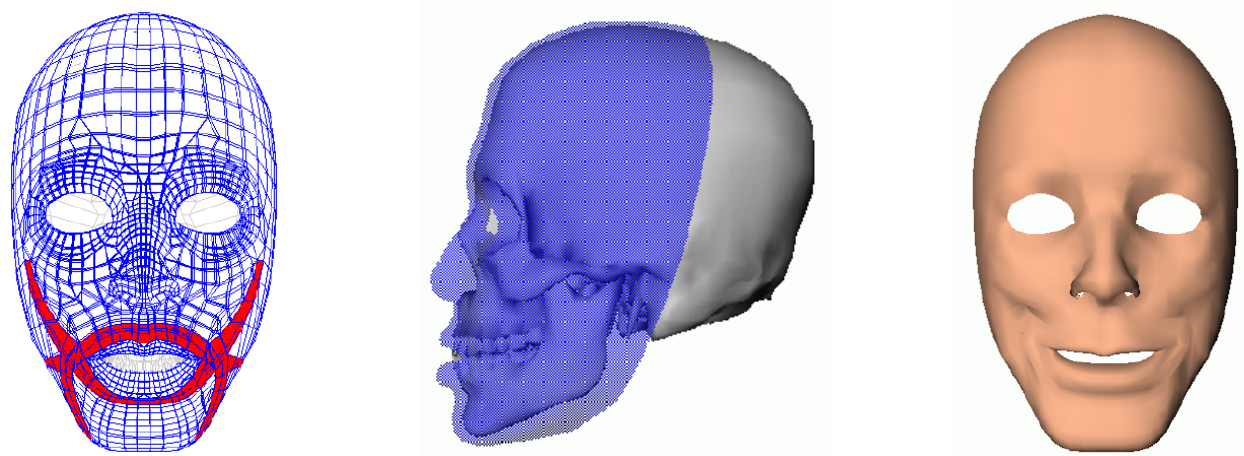

Figure 1b: Generic Finite Element model: dermis and hypodermis fixed to the skull (center), course (left) et action (right) of the muscular fibers (Chabanas et al., 2003). 

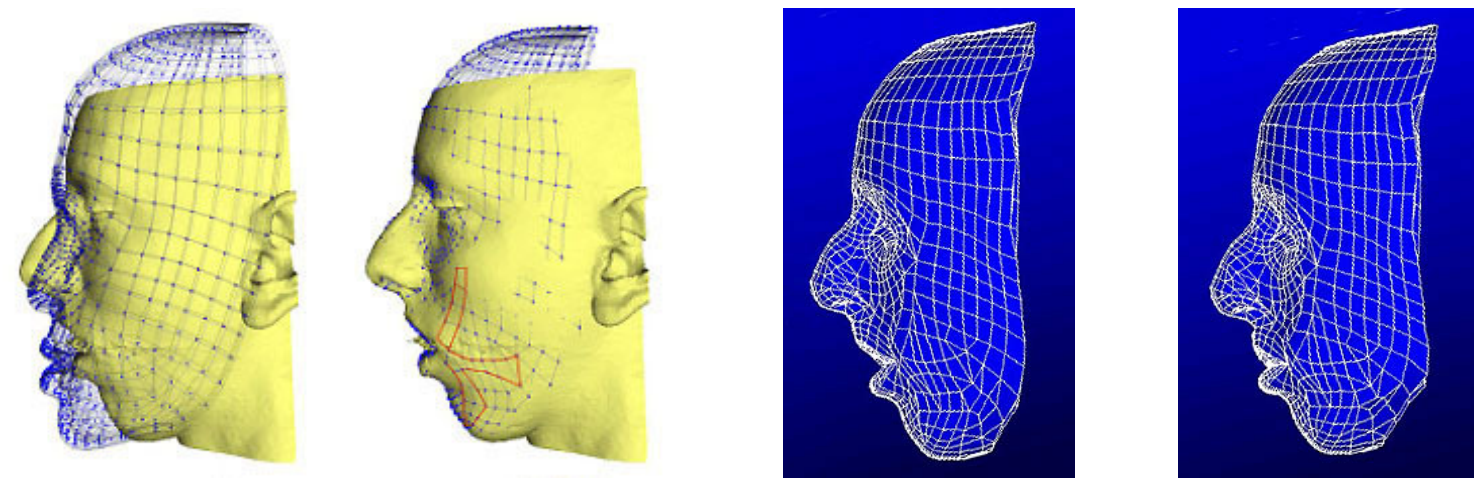

Figure 1c: Registration generic model $\rightarrow$ morphology of the patient.

Figure 1d: Predictions of the skin deformations due to the planned bone repositioning.

Left: generic model (blue) and $3 D$ reconstruction of the patient's morphology (yellow).

Right: Result of the elastic registration.
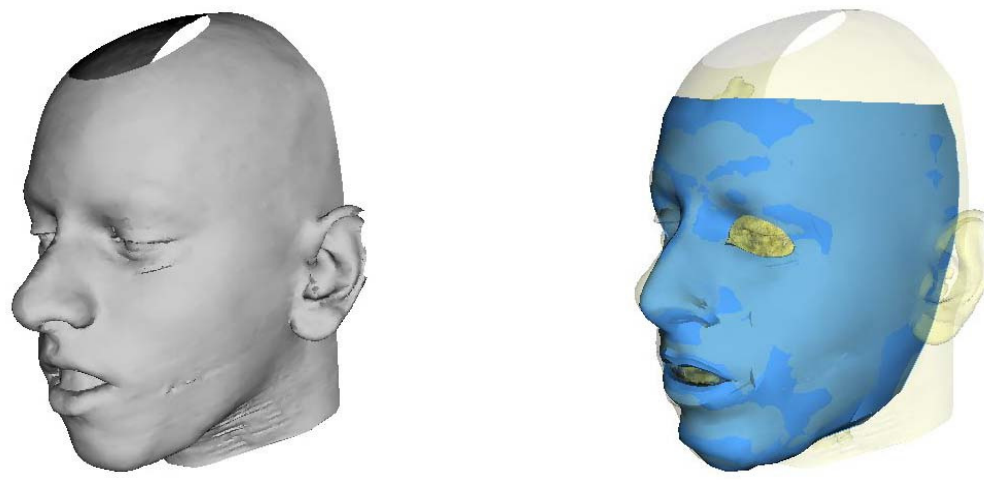

Figure 1e: Post-operative data (left) and comparison with the predictions provided by the model (right)

\section{CALIBRATION OF THE MATERIAL PROPERTIES}

The example provided in figure 1 needs the facial soft tissues material properties to be calibrated to run the simulations. This can be done assuming a mean generic values for such parameters (e.g. through ex vivo testing) or trying to fit the actual behavior of patient's soft tissues (in vivo measurements).

\subsection{Generic ex vivo calibration of the material properties}

Some years ago, our group has proposed to characterize the mechanical behavior of the human cheek. For this, an indentation experiment was provided, by measuring the mechanical response of cheek tissues removed from the fresh cadaver of a 74 year old woman (Gerard et al., 2005). The experiment was run less than $24 \mathrm{~h}$ after death, in order to limit tissue deterioration. The method 
consists in exerting a calibrated pressure onto the material and simultaneously recording the corresponding deformations (figure 2).

The indentation experiment itself does not provide the constitutive law of the material. Indeed, the measurements only give the non-linear relationship between the local force applied to the external surface of the body and the resulting displacement. To get the constitutive law from this indentation experiment, i.e. the global relationship that can be assumed between strain and stress inside the body, an optimization algorithm based on an "analysis by synthesis" strategy was elaborated. It consisted in (1) assuming a given constitutive law, (2) building a finite element analysis (FEA) of the indentation experiment, (3) comparing the simulations provided by this FEA with the indentation measurements, (4) using this comparison to propose a change of the constitutive law that should make the FEA simulations and the measurements closer, and (5) starting again with (2) up to the point where the comparison carried out in (3) gives satisfactory results.

According to Fung (1993), a hyperelastic material seems to be well adapted for human soft tissues. A material is said to be hyperelastic if it is possible to find a function $\mathrm{W}$, called the "strain-energy function", the derivative of which with respect to the strain tensor equals the stress tensor. Among the various strain-energy functions which can describe the non-linear mechanical response measured during our indentation experiment, we have proposed to use the incompressible two parameter Yeoh strain-energy function (Gerard et al., 2005). Such a constitutive law can therefore be used as an input to our ANSYS simulations to provided results such as those illustrated on figure $1 \mathrm{~d}$.

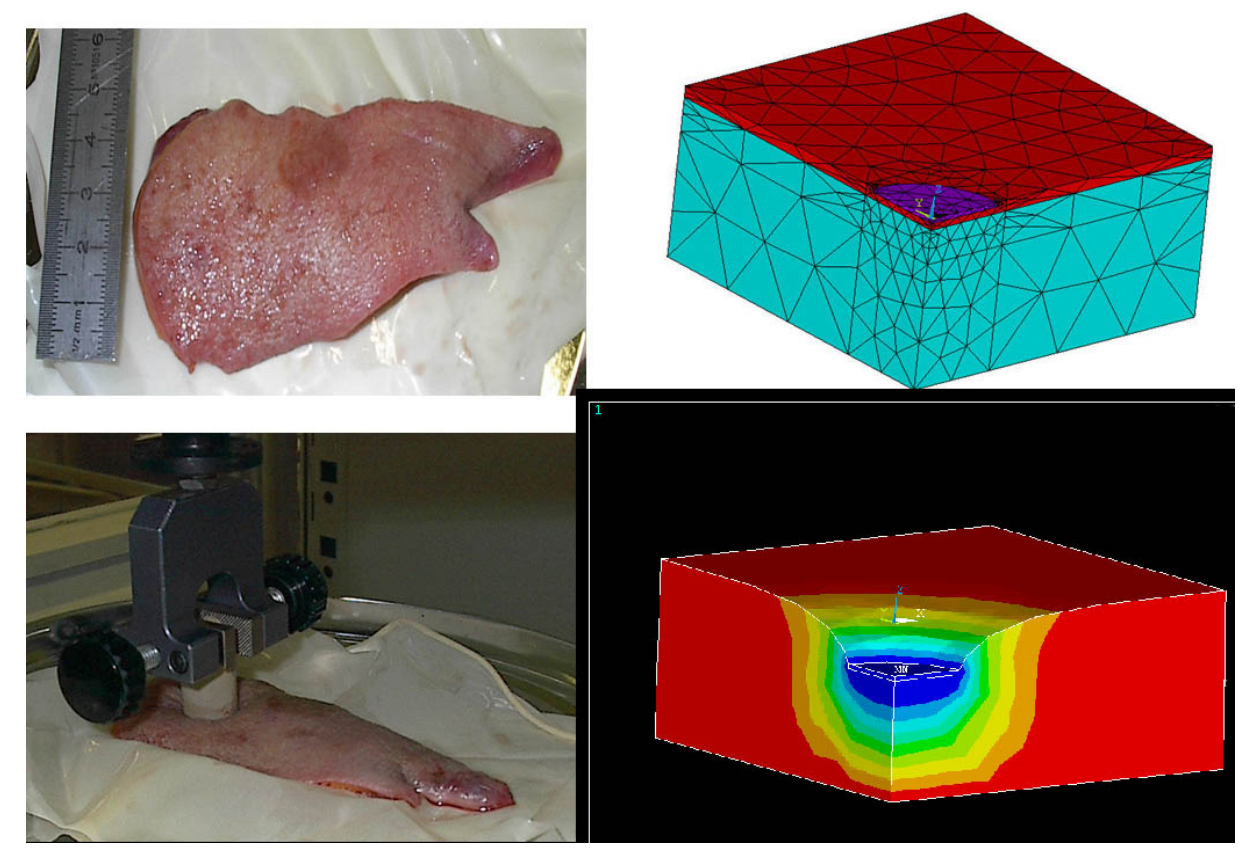

Figure 2: Indentation on a cheek specimen and the corresponding axisymmetric FE analysis 


\subsection{In vivo calibration of the material properties}

Regarding the question of mechanical properties, it is now undoubtedly established that the constitutive behaviors of human soft tissues are patient-specific and cannot be obtained by a generic value deduced from any standard ex vivo measurements. Indeed, it has clearly been shown (Kerdok et al., 2006; Ottensmeyer, 2002; Gefen and Margulies, 2004) that the mechanical behavior of soft tissue can differ significantly between in vivo and ex vivo conditions, for a number of reasons, including the vascularization of the tissue.

The various excitation methods range from suction to ultrasound with a variety of devices in each category. Aspiration is probably the most widely used technique. Starting from the pioneering work by Grahame and Holt (1969), several authors proposed suction cups differing mostly in the way the aspirated height is measured (optically Kauer et al., 2002, using ultrasound (Diridollou et al., 2000)) or by their ability to accurately measure the dynamic response. Other excitation methods include indentation (Ottensmeyer, 2001; Carter et al., 2001; Samur et al., 2007) using a handheld or robotic indenter, and torsion (Agache et al., 1980) or ballistometer (Jemec et al., 2001) which consists of striking the tissue with a known mass and a known force. Ultrasound measurement (Chen et al., 1996; Gennisson et al., 2004) is another method linked with the emerging field of elastography.

Aspiration is probably the most widespread method because it is non-invasive and proved to be quite reliable. However, most of the papers rarely address the important issue of sterilization to any great depth of detail whereas such sterilization can be very important in case of the aspiration of human living tissues. In such a perspective, our group has developed a sterilizable device (figure 3), called Light Aspiration device for in vivo Soft TIssue Characterization (for LASTIC), based on the aspiration technique and originaly introduced by Schiavone et al. (2009) while quantifying the human brain behavior. LASTIC is a $33 \times 34 \mathrm{~mm}^{2}$ metal cylinder composed of two compartments. The lower one is an airtight chamber, open at the bottom by a $10 \mathrm{~mm}$ diameter circular aperture and closed at the top by a glass window. The upper compartment holds the electronic part consisting of a miniature 2 megapixel digital camera and a LED used as a light source. The aspiration chamber is connected to a programmable syringe pump that can generate a negative pressure (measured by a manometer) which deforms the tissues on which LASTIC is laid on. This deformation is imaged by the camera via a $45^{\circ}$ inclined mirror which provides a view of the tissue from the side. The height of the tissue deformation is segmented on the recorded image. Measuring the deformation height corresponding to several steps of increasing negative pressures can give an estimation of the behavior of the tissues. Like for the indentation experiment (figure 2), the deformation/pressure measurements are then processed through an inverse Finite Element analysis to estimate the tissues constitutive laws. 


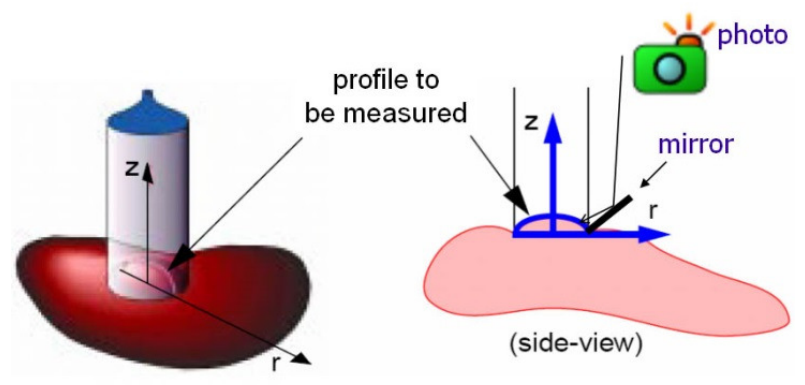

Figure $3 a:$ Principle of the in vivo aspiration method

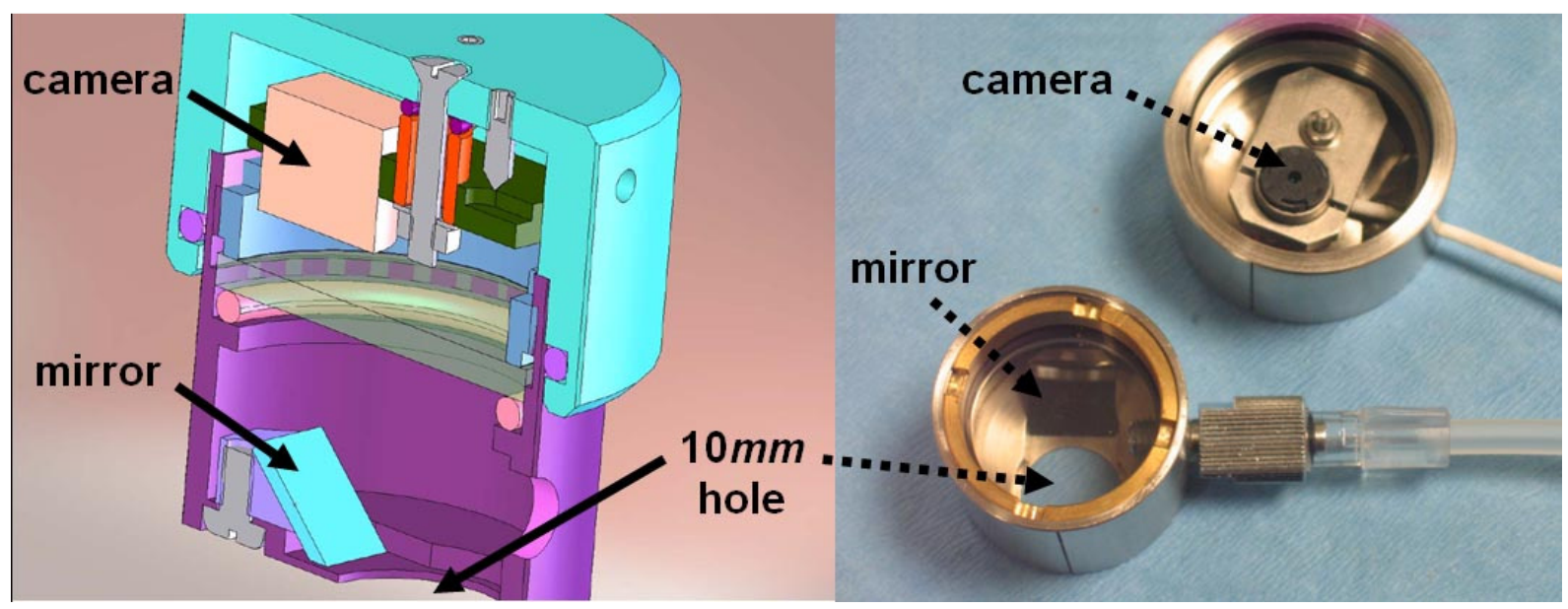

Figure 3b: LASTIC's two compartments. The lower part is the aspiration chamber with the mirror and the upper part contains the camera that images the deformation (Schiavone et al., 2010)

To be able to improve the planning of the outcome of an aesthetic or maxillo-facial surgery, it is necessary to estimate the stiffness of the facial soft tissues in several places. Four locations with presumably different tissue thicknesses and different amounts of fat and muscle were consequently chosen: the cheek, the cheekbone, the forehead, and the lower lip. To study the possible variations between subjects, the stiffness estimation was performed on a group of 16 healthy subjects, eight males and eight females, of different ages and body mass indexes. LASTIC was used to estimate the stiffness of the soft tissues at these four locations for these 16 subjects (figure 4). 

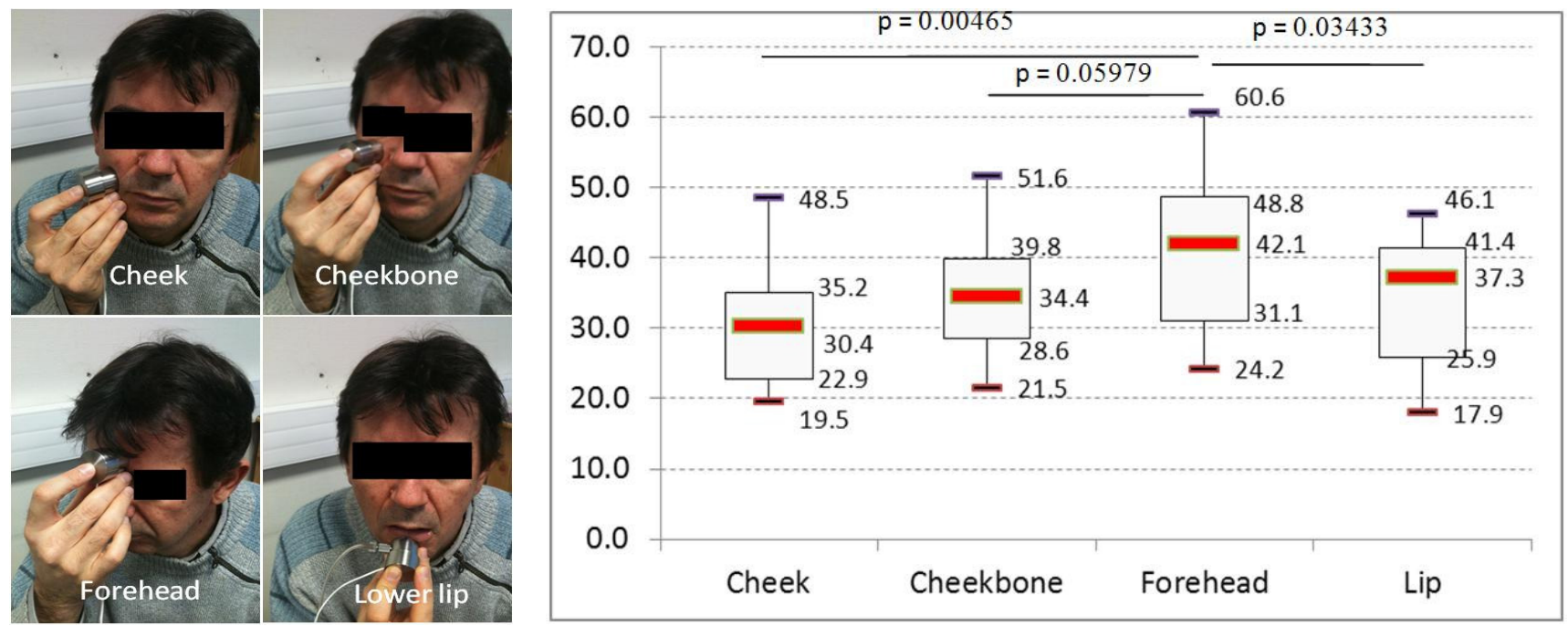

Figure 4: Facial tissues equivalent Young moduli ( $\mathrm{kPa}$ ) measured at four locations (Luboz et al., 2014)

Significant intra-subject differences in tissue stiffness are highlighted by these estimations. They also show important inter-subject variability for some locations even when mean stiffness values show no statistical difference. This study stresses the importance of using a measurement device capable of evaluating the patient specific tissue stiffness during an intervention.

The corresponding constitutive parameters can therefore be entered into our ANSYS environment to simulate face deformations due to bone repositioning. With a dedicated Usermat functionality of ANSYS, a transversely isotropic material was implemented to model muscle contraction (Nazari et al., 2011a). The activation is accounted for by adding an active stress to the Cauchy stress in the direction of fibers. An additional isochoric term is included in the strain energy function, to account for lateral normal stiffness. Figure 5 illustrates such muscle action with the deformations induced by the activation of the orbicularis oris peripheral muscle which is a sphincter muscle running around the lips and responsible for lip protrusion (Nazari et al., 2011b ; Stavness et al., 2013)). 


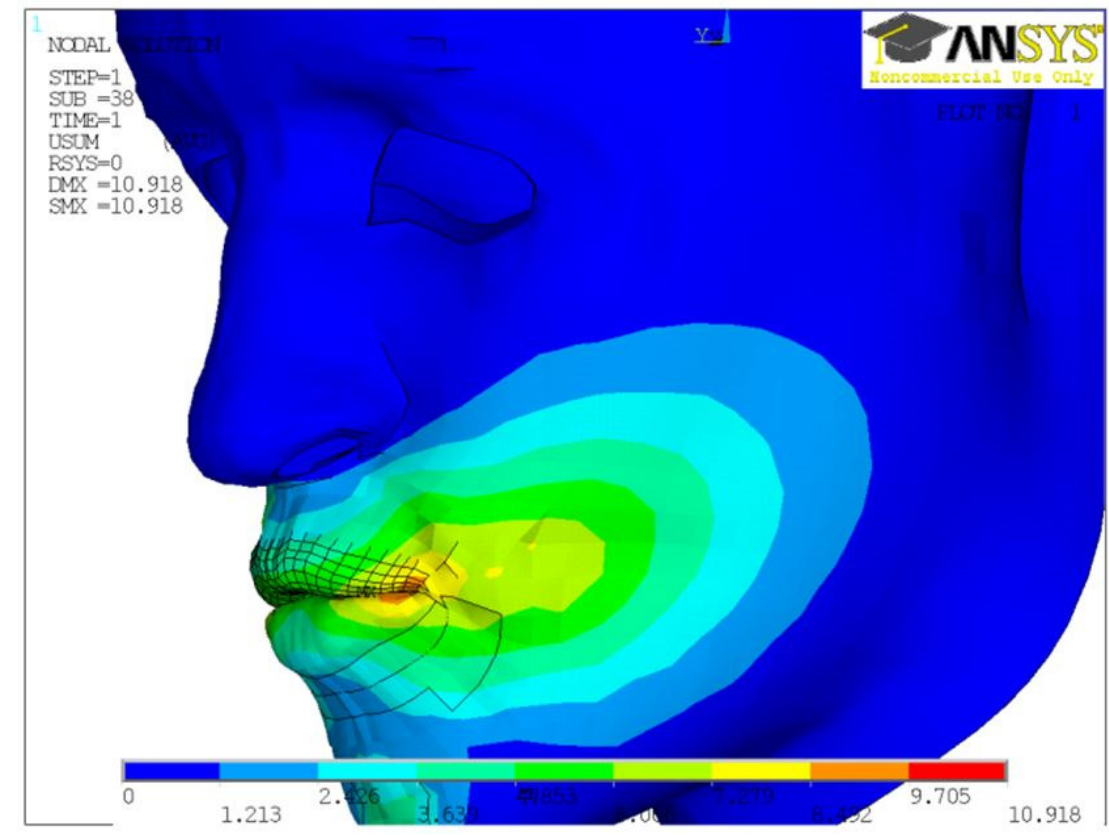

Figure 5: Face deformations due to the activation of the orbicularis oris peripheral muscle

\section{CONCLUSION}

This chapter aimed at illustrating the design of a patient-specific Finite Element model of soft tissues in a concrete clinical context. The example of the assistance of orthognathic surgery was chosen with the use of a biomechanical model of facial soft tissues including skin layers and muscular structures. The idea is to use such a model to predict the aesthetic and functional consequences of maxillary and mandible bone repositioning.

The chapter has focused on the two main difficulties in elaborating such a model, namely (1) how to generate structured patient-specific FE models in a time scale that remains compatible with the clinical constraints and (2) how to calibrate the mechanical parameters associated to these models. Ex vivo and in vivo calibrations were addressed as concerns face tissues.

However, if the constitutive laws based on such calibration processes are important, it should be acknowledged that this importance is relative if boundary conditions are not sufficiently accurately taken into account. Indeed, as was shown in Chabanas et al. (2004), changes in such boundary conditions (the displacements imposed to some nodes attached to the bony surfaces in our case) can have a much larger influence than any changes in the constitutive behaviors of facial soft tissues. This point is consistent with the recent observations of Wittek et al. (2009) who mentioned, in the 
case of a brain FE model deformed by nodes' imposed displacements, the "unimportance of constitutive models in computing brain deformation for image-guided surgery".

\section{ACKNOWLEDGMENTS}

Many scientific colleagues and clinicians have participated to the works listed in this chapter. I would like to thank them for their important inputs. Hoping that I won't forget anyone, I'd like to list Georges Bettega, Franck Boutault, Marek Bucki, Matthieu Chabanas, Jean-Michel Gerard, Vincent Luboz, Christophe Marécaux, Mohammad Nazari, Jacques Ohayon, Pascal Perrier, Emmanuel Promayon and Patrick Schiavone.

\section{REFERENCES}

Agache, P.G., Monneur, C., Leveque, J.L., Rigal, J.De., 1980. Mechanical properties and Young's modulus of human skin in vivo. Arch. Dermatol. Res. 269 (3), 221-232.

Barré, S., Fernandez, C., Paume, P., Subrenat, G., 2000. Simulating facial surgery. Proc. IS\&T / SPIE Electron. Imaging 3960, 334-34.

Bucki M., Lobos C. \& Payan Y. (2010). A Fast and Robust Patient Specific Finite Element Mesh Registration Technique: Application to 60 Clinical Cases. Medical Image Analysis, Vol. 14, pp. 303-317.

Carter, F.J., Frank, T.G., Davies, P.J., McLean, D., Cuschieri, A., 2001. Measurements and modelling of the compliance of human and porcine organs. Med. Image Anal. 5 (4), 231-236.

Chabanas M. (2002). Modélisation des tissus mous de la face pour la chirurgie orthognatique assistée par ordinateur, PhD de l’Université Joseph Fourier, Grenoble 1.

Chabanas M., Luboz V. \& Payan Y. (2003). Patient specific Finite Element model of the face soft tissue for computer-assisted maxillofacial surgery, Medical Image Analysis, Vol. 7, Issue 2, pp. 131151.

Chabanas M., Payan Y., Marécaux C., Swider P. \& Boutault F. (2004). Comparison of linear and non-linear soft tissue models with post-operative CT scan in maxillofacial surgery. Lecture Notes in Computer Science, vol. 3078, pp. 19-27. 
Chen, E.J., Novakofski, J., Jenkins, W.K., O’Brien Jr., W.D., 1996. Young's modulus measurements of soft tissues with application to elasticity imaging. IEEE Trans. Ultrason. Ferroelectr. Freq. Control 43 (1), 191-194.

Couteau B., Payan Y. \& Lavallée S. (2000). The Mesh-Matching algorithm: an automatic 3D mesh generator for finite element structures, Journal of Biomechanics, vol. 33/8, 1005-1009.

Delingette, H., Subsol, G., Cotin, S., Pignon, J., 1994. A Craniofacial Surgery Simulation Testbed. INRIA, Technical Report 2199.

Diridollou, S., Patat, F., Gens, F., Vaillant, L., Black, D., Lagarde, J.M., Gall, Y., Berson, M., 2000. In vivo model of the mechanical properties of the human skin under suction. Skin Res. Technol. 6 (4), 214-221.

Fung, Y.C., 1993. Biomechanics: Mechanical Properties of Living Tissues. Springer, New York.

Gefen A., Margulies S., 2004. Are in vivo and in situ brain tissues mechanically similar? J. Biomech. 37 (9), 1339-1352.

Gennisson, Jean-Luc, Baldeweck, Thérèse, Tanter, Mickaël, Catheline, Stefan, Fink, Mathias, Sandrin, Laurent, Cornillon, Céline, Querleux, Bernard, 2004. Assessment of elastic parameters of human skin using dynamic elastography. IEEE Trans. Ultrason. Ferroelectr. Freq. Control 51 (8), 980-989.

Gerard J.M., Ohayon J., Luboz V., Perrier P. \& Payan Y. (2005). Non linear elastic properties of the lingual and facial tissues assessed by indentation technique. Application to the biomechanics of speech production, Medical Engineering \& Physics, Vol. 27 (10), pp. 884-892.

Gladilin, E., Zachow, S., Hege, H.C., Deuflard, P., 2001. FE-based heuristic approach for the estimation of person-specific facial mimics. In: Middleton, I., Jones, M.L., Shrive, N.G. (Eds.), $5^{\text {th }}$ International Symposium on Computer Methods in Biomechanics and Biomedical Engineering, Rome, Italy, November 2001.

Grahame, R., Holt, P.J., 1969. The influence of ageing on the in vivo elasticity of human skin. Gerontologia 15 (2), 121-139.

Jemec, G.B., Selvaag, E., Agren, M., Wulf, H.C., 2001. Measurement of the mechanical properties of skin with ballistometer and suction cup. Skin Res. Technol. 7 (2), 122-126.

Kauer, M., Vuskovic, V., Dual, J., Szekely, G., Bajka, M., 2002. Inverse finite element characterization of soft tissues. Med. Image Anal. 6 (3), 275-287. 
Keeve, E., Girod, S., Girod, B., 1996. Computer-aided craniofacial surgery. In: Lemke, H.U. et al. (Ed.), Computer Assisted Radiology, Elsevier, pp. 757-763.

Keeve, E., Girod, S., Kikinis, R., Girod, B., 1998. Deformable modelling of facial tissue for craniofacial surgery simulation. Computed Aided Surgery, 3 (5), 228-238.

Kerdok, Amy E., Ottensmeyer, Mark P., Howe, Robert D., 2006. Effects of perfusion on the viscoelastic characteristics of liver. J. Biomech. 39 (12), 2221-2231.

Koch, R.M., Gross, M.H., Carls, F.R., von Büren, D.F., Frankhauser, G., Parish, Y.I.H., 1996. Simulating facial surgery using finite element models. Proceedings of SIGGRAPH. ACM Press, pp. 421-428.

Koch, R.M., Roth, S.H.M., Gross, M.H., Zimmermann, A.P., Sailer, H.F., 1999. A framework for facial surgery simulation. CS Technical Report \#326, 18 June 1999. ETH Zurich, Switzerland.

Luboz V., Promayon E. \& Payan Y. (2014). Linear Elastic Properties of the Facial Soft Tissues Using an Aspiration Device: Towards Patient Specific Characterization. Annals of Biomedical Engineering, Vol. 42 (1), pp. 2369-2378. DOI: 10.1007/s10439-014-1098-1.

Mollemans, W, Schutyser, F, Nadjmi ,N, Maes, F., \& Suetens, P. (2007). Predicting soft tissue deformations for a maxillofacial surgery planning system: From computational strategies to a complete clinical validation, Medical Images Analysis, 11, 282-301.

Nazari M., Perrier P., Chabanas M. \& Payan Y. (2010). Simulation of dynamic orofacial movements using a constitutive law varying with muscle activation. Computer Methods in Biomechanics \& Biomedical Engineering, Vol. 13(4), pp. 469-482.

Nazari M.A., Perrier P., Chabanas M. \& Payan Y. (2011a). A 3D Finite Element Muscle Model and its Application in Driving Speech Articulators. Proceedings of the 23rd Congress of the International Society of Biomechanics, ISB'2011, Brussels, Belgium.

Nazari M., Perrier P., Chabanas M. \& Payan Y. (2011b). Shaping by stiffening: a modeling study for lips. Motor Control, Vol. 15(1), pp. 141-168.

Ottensmeyer, Mark Peter., 2001. Minimally Invasive Instrument for in vivo Measurement of Solid Organ Mechanical Impedance. Ph.D. Thesis, Massachusetts Institute of Technology, Dept. of Mechanical Engineering.

Ottensmeyer M., 2002. In vivo measurement of solid organ visco-elastic properties. Stud. Health Technol. Inform. 85, 328-333. 
Richter M., Mossaz C., Laurent F., Goudot P. Chirurgie correctrice des malformations maxillomandibulaires : approche diagnostique et plan de traitement. Encycl. Méd. Chir. (Elsevier, Paris), Stomatologie, 22-066-D-10, 1998, 16p. 1998.

Rouviere, H., Delmas, A. (1997). In: Tete et Cou, 14th Edition. Anatomie Humaine Descriptive, Topographique et Fonctionnelle, Vol. 1. Masson, Paris.

Samur, E., Sedef, M., Basdogan, C., Avtan, L., Duzgun, O., 2007. A robotic indenter for minimally invasive measurement and characterization of soft tissue response. Med. Image Anal. 11 (4), 361373.

Schiavone P., Chassat F., Boudou T., Promayon E., Valdivia F. \& Payan Y. (2009). In vivo measurement of human brain elasticity using a light aspiration device. Medical Image Analysis, Vol. 13, pp. 673-678.

Schiavone P., Promayon E. \& Payan Y. (2010). LASTIC: a Light Aspiration device for in vivo Soft TIssue Characterization. Lecture Notes in Computer Science, Vol. 5958, pp. 1-10.

Stavness I., Nazari M., Perrier P., Demolin D. \& Payan Y. (2013). A Biomechanical Modeling Study of the Effects of the Orbicularis Oris Muscle and Jaw Posture on Lip Shape. Journal of Speech, Language, and Hearing Research, Vol. 56, pp. 878-890.

Teschner, M., Girod, S., Girod, B., 1999. Optimization approaches for soft-tissue prediction in craniofacial surgery simulation. In: 2nd International Conference on Medical Image Computing and Computer Assisted Intervention, MICCAI'99. LNCS, Vol. 1679. Springer, pp. 1183-1190.

Teschner, M., 2001. Direct Computation of Soft-tissue Deformation in Craniofacial Surgery Simulation. Ph.D. Thesis, Shaker Verlag, Aachen, Germany (January 2001).

Waters, K., 1996. Synthetic muscular contraction on facial tissue derived from computer tomography data. In: Taylor, R., Lavallee, S., Burdea, G., Mosges, R. (Eds.), Computer Integrated Surgery. MIT Press, Cambridge, MA, pp. 191-200.

Wittek A., Hawkins T. \& Miller K. (2009). On the unimportance of constitutive models in computing brain deformation for image-guided surgery. Biomech Model Mechanobiol., Vol 8(1), pp. $77-84$.

Zachow, S., Gladiline, E., Hege, H.-C., Deuflhard, P., 2000. Finite element simulation of soft tissue deformation. In: Proceedings of CARS, pp. 23-28. 